\title{
Detectives in the laboratory: investigation of a seemingly unexplained error
}

\author{
Massimo De Paschale, ${ }^{1}$ Sergio Finazzi, ${ }^{2}$ Gianpietro Re, ${ }^{2}$ Milvia Lotzniker, ${ }^{2}$ Pierangelo Clerici1 \\ ${ }^{1}$ Microbiology Unit, and ${ }^{2}$ Clinical Chemistry Laboratory, Hospital of Legnano, ASST Ovest Milanese, Legnano, Italy
}

\section{Summary}

An unexplainable error that occurred in the normal laboratory routine is described: a positive result for the anti-HCV antibodies detection was found in a single blood tube collected from a patient, while the other samples taken simultaneously were antiHCV negative. After the first checks that did not highlight any special problems, an internal committee composed of the chiefs of the units involved was established. This committee, using investigative analysis with the Root Causes Analyses model was able to understand the error.

\section{Introduction}

Errors in medicine laboratory may regard each of the main phases of technical procedures: pre-analytical phase (before the arrival of the sample), analytical and post-analytical phase (after issuing the report). On the total number of errors, the literature reports that only $7-13 \%$ is due to errors in the analytic phase, while $46-68 \%$ is due to errors in the pre-analytical phase and 18.5 $47 \%$ in the post-analytical phase $(3,8,25)$.

In the pre-analytical phase, the main problems relate to inappropriate tests request (23), patient and specimen misidentification $(1,10,22)$, inadequacy of the sample (hemolysed or curdled sample, insufficient or inappropriate quantities, wrong container,

Correspondence: Massimo De Paschale, Microbiology Unit, Hospital of Legnano, ASST Ovest Milanese, Via Papa Giovanni Paolo II, 20025 Legnano (MI), Italy.

E-mail: massimo.depaschale@asst-ovestmi.it

Key words: Medical error; Laboratory.

Contributions: the authors contributed equally.

Conflict of interest: the authors declare no potential conflict of interest.

Received for publication: 12 January 2017

Accepted for publication: 15 March 2017.

(C) Copyright M. De Paschale et al., 2017

Licensee PAGEPress, Italy

Microbiologia Medica 2017; 32:6572

doi:10.4081/mm.2017.6572

This article is distributed under the terms of the Creative Commons Attribution Noncommercial License (by-nc 4.0) which permits any noncommercial use, distribution, and reproduction in any medium, provided the original author(s) and source are credited. etc.) $(7,17)$ and handling, storage and transport of the sample to the laboratory (19). In the post-analytical phase, the most encountered problems concern the denied or delayed computer accessibility to the test results (11) and incorrect interpretation of the laboratory report (19). In the analytical phase, problems regard the different stages of the procedures in the laboratory: in the first instance, the sample preparation (centrifugation, aliquotting, pipetting, dilution and sorting specimen into batches for their introduction into automated analyzers) was extensively evaluated as an important source of error (19). The availability of instruments using primary collection tubes and the introduction of automated work stations for the sample preparation drastically reduced these errors (5). Among the errors in the analytical phase, however, those related to the contamination (by other samples) (4) and to interference from different substances present in the same sample (heterophilic antibodies, anti-animal antibodies, autoantibodies, etc.) were extensively investigated $(9,14,19)$. Even the analytical accuracy was widely discussed in the literature and, for example, the impact of calibration errors was carefully considered, with regard to the negative consequences that may have on clinical outcome $(12,16)$. The wrong validation, the delay in reporting and the transcription errors were also reported $(2,18)$. The automatic validation process (19) and the computerized final report without manual transcription minimized the errors. But, where there are still manual methods (typically in bacteriology), the error of transcription is still widely possible.

Although standardization of methods, advanced automation of procedures and technological improvements greatly improved the reliability with a substantial decrease of the errors (24), errors can nevertheless occur. Given the importance of errors in healthcare, documents on Risk Management were produced that consider both human behavior as a source of error (aberrant behavior), and the conditions (technological, relational and human factors) in which the error occurred, all seen as the outcome of a system failure (15). Effective activity of Risk Management regards the knowledge and error analysis, the detection and correction of error causes, and the monitoring of implemented measures for error prevention (15). In order to implement structural survey, able to recognize the causes that have produced the error, a reactive analysis (15) which is conducted backwards compared to the timeline of error, can be used. For this purpose, the Root Causes Analyses (RCA) can be implemented. These analyses, starting from the errors found in a system, are suitable to look for causes through an inductive method which proceeds in depth with questions that explore the why of every action and each of its possible deviation (6). This activity is not always easy to take, especially if the error occurred is acknowledged at a distance of time. Moreover, the complexity of the operations performed in the laboratory does not always allow a quick identification of the critical point. We describe a seemingly inexplicable error occurred in the normal laboratory routine after which we used a RCA, which led to fully understanding the error. 


\section{Description of the error}

A hospital employee came to the Blood Drawing Center for testing her 3-year-old daughter. Blood chemistry and infectivological tests were prescribed by the general practitioner treating the child for unspecific symptoms of malaise. The number of primarytest tubes for the different tests was high: one tube for blood count (sent to the Blood Transfusion Centre), one for biochemical tests, one for electrophoresis and one for autoantibodies (all three sent to Clinical Chemistry Laboratory) and one for infectivological tests (anti-CMV, anti-EBV, anti-Adenovirus etc. sent to the Microbiology Laboratory). The woman and a physician of the Microbiology Laboratory, agreed to add more tests in case of negativity of the first infectivological tests. Given this possibility, as the amount of blood in the tubes was little, being the child very young, it was requested to keep as many blood tubes as possible. A primary-tube (in this case the one for electrophoresis) was then immediately taken back from the Clinical Chemistry Laboratory and stored in a refrigerator of the Microbiology Laboratory. As the different infectivological tests were negative, the physician of the Microbiology Laboratory requested to add tests for the markers of viral hepatitis, including anti-HCV. These tests were then performed on the electrophoresis tube because this tube contained more blood than others. The results for markers of hepatitis were all negative except for anti-HCV. Despite a value not particularly high, it was also positive after execution of the confirmation test in immunoblot. Being the woman anti-HCV negative, and being her daughter known by the laboratory staff, the question of the possible route of infection was immediately raised. Since the risk factors for HCV were not known for the child, it was decided to review the results of anti-HCV search also on the blood remained in the primary-tube for anti-CMV and anti-EBV (still stored in the Microbiology Laboratory) and in the secondary-tube for autoantibodies (stored in the Clinical Chemistry Laboratory). On both tubes the result for anti-HCV was negative. While the tube for autoantibodies was a secondary-tube (after separation of the sam$\mathrm{ple}$ ), the tube for anti-CMV/EBV and the one for electrophoresis were primary-tubes. Given that one of primary-tubes (for anti$\mathrm{CMV} / \mathrm{EBV}$ ) was anti-HCV negative and one (for electrophoresis) anti-HCV positive, both tubes having been processed directly in the analyzer for anti-HCV, the discrepancy was not due to the manipulation of the tubes. The little girl was then redrawn, and the negativity for anti-HCV was confirmed. Following the seemingly inexplicable inconsistency of such data, an internal investigation conducted in collaboration between qualified staff from the Microbiology Laboratory, Clinical Chemistry Laboratory and Blood Drawing Center was initiated.

\section{Conducted survey}

Since it was established that the little girl was negative for anti$\mathrm{HCV}$, attention was focused on the tube that had repeatedly given the positive result.

The first hypothesis taken into account, was an exchange of tubes at the time of collection (error during pre-analytical phase). The survey carried out with the Blood Drawing Center staff did not, however, show abnormalities. All the test-tubes had been automatically labeled by the computer system of the Center. The collection had been properly performed by the nursing staff and there had been no situation of confusion at the time of sampling or particular anomalies (e.g. change at the last minute of a test tube for difficulty of collection, or the like). The mother was present with the child in the withdrawal box, she saw all tubes correctly labeled and the nurse, having checked the identity of the girl, collected the blood with no difficulties. The sampling was performed only to the girl and not to the mother, so the hypothesis of a yet possible exchange with the mother was rejected (by the way, the mother was known to be negative for anti-HCV). In addition, the subsequent verification of the primary-tubes did not show any overlapping label. Moreover, it is impossible that a label is attached on a unlabeled full blood tube (of a different patient) in that the blood collection is performed only on pre-labeled tubes). The nurse who performed the sampling denied that at the time there were other anomalies.

As a second hypothesis, the possibility of a contamination of the tube with anti-HCV positive result (error in analytical step), was taken into account; in the Microbiology Laboratory, the tube was inserted uncorked into the analyzer for automatic execution of tests (as in all normal routine) without further manipulations. For the draft of the sample the analyzer (as for other analytical steps) used disposable tips. The tube came from the Clinical Chemistry Laboratory where it had been processed for electrophoresis and then delivered to the Microbiology Laboratory. Also in this case the tube, uncorked, was inserted into the electrophoresis analyzer, which uses for drafting a high-washing fixed needle to avoid phenomena of carry over.

In the absence of satisfactory explanations, it was decided to follow the entire route of the suspected tube, trying to stimulate all the involved technicians to remember every unusual detail, even the smallest, about what had happened. As a result, the technician of Microbiology Laboratory who had performed the analysis for anti$\mathrm{HCV}$, recalled that she had noticed that the tube for electrophoresis was capped with the original cap. She had, therefore, thought the tube was not used or that the electrophoresis system (she did not know it because the system belong to a different Laboratory) would use a needle piercing the original cap (as with blood count tests). The graduate of the Clinical Chemistry Laboratory rejected this hypothesis because the electrophoresis analyzer uses uncorked tubes. Normally the tubes are then re-corked with new caps that are different from those of the original tube.

Further investigation therefore focused on the procedures at the time of electrophoresis. The technician who had performed the electrophoresis, remembered to have uncorked the tube, performed electrophoresis and finally to have given the tube to an attendant to be delivered to the Microbiology Laboratory, but to have corked it hastily with one of the caps of the uncorked tubes waiting for electrophoresis routine.

In conclusion, the tube was not re-corked with a clean cap, nor with the original cap, but with a cap belonging to a test tube of another patient, which explains the contamination.

\section{Conclusions}

In front of unexplained errors, it is often difficult to understand the dynamics, partly because they manifest themselves after a long time when it is more complicated, if not impossible, to reconstruct all the events. In our case, in fact, only the knowledge of the patient immediately raised doubts about the test result and allowed the reconstruction of the events in a short time. The establishment of a committee that involved all the implicated Units, allowed us a 360 -degrees investigation and to set up a methodology that resulted in the understanding of the error.

In this case the error was human. According to Rasmussen (20), human behavior can be divided into three different types: skill-based behavior (automatic behavior to a given situation), 
ruled-based behavior (implementation of practices prescribed by rules) and knowledge-based behavior (behaviors adopted when man is faced with an unfamiliar situation). Based on this model, Reason (21) identified three types of error: execution errors caused by a memory failure (lapses), errors committed during the practical execution of the action (mistakes) and execution errors that occur in skill level (slips). In this last category all actions that are performed in a way other than planned are classified, that is, the subject knows how to behave but it does not, or inadvertently runs incorrectly.

What had happened was what Reason calls violation (21), that is a deviation from the written procedures. Starting from the consideration that error is an inevitable part of human reality (13), it is crucial to recognize the circumstances that favored the occurrence of the error to put in place a set of actions that make it more difficult for people to repeat mistakes. In our case, the deviation from normal codified procedures for the proper management of samples of a known patient to whom particular attention had been paid, consisted of the technician's acting hastily and superficially, so the slip took place at the level of skill-based behavior. The rest, it is common sense that the inconveniences take place always to known people to which favors are done, where the input to the change of an automatic behavior can lead to an error.

To conclude, by gathering the resources of all the involved staff, a proper investigation was carried out, which led to understand the specific mistake and to implement appropriate solutions capable of preventing the perpetuation of errors.

\section{References}

1. Altman DE, Clancy C, Blendon RJ. Improving patient safety-five years after the IOM report. N Engl J Med 2004;351:20413.

2. Astion ML, Shojania KG, Hamill TR, et al. Classifying laboratory incident reports to identify problems that jeopardize patient safety. Am J Clin Pathol 2003;120:18-26.

3. Boone DJ. Is it safe to have a laboratory test? Accred Qual Assur 2004;10:5-9.

4. Busch MP, Kleinman SH, Nemo GJ. Current and emerging infectious risks of blood transfusions. JAMA 2003;289:959-62.

5. Holman JW, Mifflin TE, Felder RA, Demers LM. Evaluation of an automated preanalytical robotic workstation at two academic health centers. Clin Chem 2002;48:540-8.

6. The Joint Commission. Accreditation of Healthcare Organization. Available from: http://www.jcaho.org/ptsafety_ frm.html

7. Jones BA, Calam RR, Howanitz PJ. Chemistry specimen accept- ability: a College of American Pathologists Q-Probes study of 453 laboratories. Arch Pathol Lab Med 1997;121:19-26.

8. Kalra J. Medical errors: impact on clinical laboratories and other critical areas. Clin Biochem 2004;37:1052-62.

9. Kazmierczak SC, Catrou PG. Analytical interference. More than just a laboratory problem. Am J Clin Pathol 2000;113:9-11.

10. Khoury M1, Burnett L, Mackay MA. Error rates in Australian chemical pathology laboratories. Med J Aust 1996;165:128-30.

11. Kilpatrick ES, Holding S. Use of computer terminals on wards to access emergency test results: a retrospective audit. BMJ 2001;322:1101-3.

12. Klee GG, Schryver PG, Kisabeth RM. Analytic bias specifications based on the analysis of effects on performance of medical guidelines. Scand J Clin Lab Invest 1999;59:509-12.

13. Kohn L, Corrigan J, Donaldson. To err is human: building a safer health system. Washington DC: National Academy Press; 1999.

14. Marks V. False-positive immunoassay results: a multicenter survey of erroneous immunoassay results from assays of 74 analytes in 10 donors from 66 laboratories in seven countries. Clin Chem 2002;48:2008-16.

15. Ministero della salute. Risk management in sanità. Il problema degli errori. Commissione Tecnica sul Rischio Clinico (DM 5 marzo 2003). Roma, Marzo 2004.

16. National Institute of Standards and Technology. Planning Report 04-1: The impact of calibration error in medical decision making. Available from: www.nist.gov/director/proofc/report04.pdf

17. Plebani M, Bonini P. Interdepartmental cooperation may help avoid errors in medical laboratories. Br Med J 2002;324:423-4.

18. Plebani M, Carraro P. Mistakes in a stat laboratory: types and frequency. Clin Chem 1997;43:1348-51.

19. Plebani M. Errors in clinical laboratories or errors in laboratory medicine? Clin Chem Lab Med 2006;44:750-9.

20. Rasmussen J, Duncan K, Leplat J. New technology and human error. Chichester: Wiley; 1987.

21. Reason J. Human error. New York: Cambridge University Press; 1990.

22. Renner SW, Howanitz PJ, Bachner P. Wristband identification error reporting in 712 hospitals. A College of American Pathologists' Q-Probes study of quality issues in transfusion practice. Arch Pathol Lab Med 1993;117:573-7.

23. Silverstein MD. An approach to medical errors and patient safety in laboratory services. A white paper. The Quality Institute Meeting, Atlanta, GA 2003.

24. Stankovic AK. The laboratory is a key partner in assuring patient safety. Clin Lab Med 2004;24:1023-35.

25. Stroobants AK, Goldschmidt HM, Plebani M. Error budget calculations in laboratory medicine: linking the concepts of biological variation and allowable medical errors. Clin Chim Acta 2003;333:169-76. 